\title{
REFLUENT MULTIFUNCTIONS ON SEMITREES
}

\author{
T. B. MUENZENBERGER AND R. E. SMITHSON
}

\begin{abstract}
The concept of refluent on arcs is introduced for multifunctions on semitrees. This notion is then used to obtain a fixed point structure which has as corollaries some generalizations of known fixed point theorems in arcwise connected spaces. For example, we generalize: each continuous point closed multifunction on an arboroid has a fixed point. The final section of the paper develops the relationships between a number of classes of multifunctions on nested spaces.
\end{abstract}

1. Introduction. In [8], L. E. Ward, Jr. proved a fixed point theorem for continuous point closed multifunctions, and in [9] he characterized dendroids in terms of the fixed point property for continuum valued upper semicontinuous multifunctions. Then in [7] Smithson generalized the fixed point theorem in [9], as well as a theorem of G. S. Young, Jr. [10], to a class of multifunctions which preserved arcwise connectedness. Recently, B. En-Nashef proved [6] a fixed point theorem for a class of multifunctions which included both of the classes studied by Ward. In [3] Muenzenberger and Smithson introduced the concept of a fixed point structure and defined the notion of a semitree. Using these ideas and the inherent partial order structure, they obtained theorems of Smithson [7], Ward [9], and Young [10] as corollaries to their main results. The purpose of the present paper is to define a fixed point structure which will subsume Nashef's theorem [6] as well as many of the other known theorems. Furthermore, it will be shown that the class of multifunctions considered here is strictly larger than the class of multifunctions considered by Nashef. Finally, a number of other relationships between certain classes of multifunctions will be obtained as well as a characterization of certain spaces by means of the fixed point property.

2. Preliminaries. Let $X$ and $Y$ be nonempty sets. A multifunction $F: X \rightarrow Y$ is a point to set correspondence (that is, $F(x)$ is a nonempty subset of $Y$ for each $x \in X$ ). If $Y$ is a topological space, the multifunction $F$ is said to be point closed (point compact, point connected, continuum

Received by the editors June 25, 1973.

AMS (MOS) subject classifications (1970). Primary 54H25.

Key words and phrases. Multifunctions, fixed point structures, dendroids, arboroids, semitrees.

(c) American Mathematical Society 1974 
valued) in case $F(x)$ is closed (compact, connected, a subcontinuum of $Y$ ) for each $x \in X$. When both $X$ and $Y$ are topological spaces, we say that $F$ is upper semicontinuous (lower semicontinuous) if and only if whenever $A \subset Y$ is closed (open), the set $F^{-1}(A)=\{x \in X: F(x) \cap A \neq \varnothing\}$ is closed (open). A multifunction which is both upper (u.s.c.) and lower (l.s.c.) semicontinuous is said to be continuous. If $A \subset X$, then

$$
F(A)=\bigcup\{F(x): x \in A\} .
$$

A fixed point of $F$ is a point $x \in X$ such that $x \in F(x)$.

Nashef used the following condition in [6].

Definition. A multifunction $F: X \rightarrow Y$ from a topological space $X$ into a topological space $Y$ is called refluent if and only if for each subcontinuum $K$ of $X$ and each component $C$ of $F(K), F(x) \cap C \neq \varnothing$ for all $x \in K$.

Nashef observed [6] that any continuum valued u.s.c. multifunction and any continuous point compact multifunction are refluent [8].

In the following an arc is a continuum which contains exactly two noncutpoints called the endpoints of the arc. A topological space is arcwise connected in case each pair of distinct points are the endpoints of some arc in the space. A space is hereditarily unicoherent whenever $A$ and $B$ are closed connected subsets of the space, $A \cap B$ is connected. An arboroid is an arcwise connected hereditarily unicoherent continuum. A dendroid is a metric arboroid. Finally, an arcwise connected space is nested in case the union of each nest of arcs in the space is contained in an arc in the space. Nested spaces were introduced by Young [10] and have been studied by several authors [2], [5]. The term arboroid is due to Ward. D. P. Bellamy [1] has shown that each subcontinuum of an arboroid is decomposable.

The characterization of a semitree as a certain kind of partially ordered set is the most convenient for the purposes of this paper. (See [3], [4], [5] for further information on semitrees.) Thus a semitree is a partially ordered set $(X, \leqq)$ which satisfies:

(i) there is a least element $e \in X$;

(ii) if $x<y$, then there is a $z \in X$ such that $x<z<y$ (that is, $\leqq$ is order dense);

(iii) for each $x \in X$, the set $L(x)=\{y \in X: y \leqq x\}$ is totally ordered;

(iv) if $\varnothing \neq A \subset X$, then inf $A \in X$;

(v) if $\varnothing \neq A \subset X$ is totally ordered, then $\sup A \in X$.

We state (i) to be explicit. If $x \leqq y$, we set $[x, y]=\{z \in X: x \leqq z \leqq y\}$. If $x$ and $y$ are not comparable, we set $[x, y]=[x \wedge y, x] \cup[x \wedge y, y]$ where $x \wedge y=\inf \{x, y\}$. Further, we define $[x, y),(x, y]$, and $(x, y)$ in the usual way; for example, $[x, y)=\{z \in X: x \leqq z<y\}$. If $x$ and $y$ are comparable, 
then $x \vee y=\sup \{x, y\}$. If $x \in X$, set $M(x)=\{y \in X: x \leqq y\}$. Then $[x, y]=$ $M(x) \cap L(y)$ if $x \leqq y$. The order topology $\mathscr{I}$ for $X$ is the topology which has all of the sets $L(x)$ and $M(x)$ for $x \in X$ as a subbase for the closed sets. Note that the sets $[x, y]$ are arcs when given the subspace topology induced by the order topology [5], [8]. Finally, a chain component of a set $A \subset X$ is a set $C \subset A$ which is maximal with respect to the property: $x, y \in C$ implies that $[x, y] \subset C$.

3. The main theorem. Now let $(X, \leqq)$ be a semitree. We shall need the following definition.

Definition. A subset $A \subset X$ is said to be closed [3], [5] in case for each totally ordered set $A_{0} \subset A$, both of inf $A_{0}$ and sup $A_{0}$ are in $A$.

Next let $F: X \rightarrow X$ be a multifunction on $X$ into $X$ and consider the following three conditions on $F$ :

(1) $F$ is refluent on arcs if and only if for each $\operatorname{arc}[x, y]$ in $X$ with $x \leqq y$ and for each chain component $C$ of $F[x, y], F(z) \cap C \neq \varnothing$ for all $z \in[x, y]$;

(2) $F(x)$ is closed for all $x \in X$;

(3) $F$ is closed under suprema if and only if whenever $\left\{x_{\alpha}: \alpha \in \mathscr{A}\right\}$ and $\left\{y_{\alpha}: \alpha \in \mathscr{A}\right\}$ are two totally ordered subsets of $X$ satisfying, $\left\{x_{\alpha}\right\}$ is increasing and $y_{\alpha} \in F\left(x_{\alpha}\right)$ for each $\alpha \in \mathscr{A}$, then $y_{0} \in F\left(x_{0}\right)$ where $x_{0}=\sup _{\alpha} x_{\alpha}$ and $y_{0}$ is any $\mathscr{I}$-cluster point of $\left\{y_{\alpha}\right\}$.

Notice that (3) implies part of (2).

If $\mathscr{P}$ denotes the set of arcs in $X$ (i.e., $\mathscr{P}=\{[x, y]: x, y \in X\}$ ), and if $\mathscr{F}$ is a set of multifunctions on $X$ into $X$ satisfying (1), (2), and (3), then we shall show that $(X, \mathscr{P}, \mathscr{F})$ is a fixed point structure as defined in [3]. To do this we must show that each member of $\mathscr{F}$ has a fixed point.

Our proof was motivated by the proof Ward gave of the main result in [8]. So let $F: X \rightarrow X$ be a multifunction and define the set $J$ as follows:

$$
\begin{array}{r}
J=\left\{x \in X \text { : there is a minimal element } t_{x} \in F(x) \cap M(x)\right. \\
\text { and if } \left.x<p \leqq t_{x} \text {, then } F(p) \cap\left[p, t_{x}\right]=\varnothing\right\} .
\end{array}
$$

The set $J$ is the set of elements in $X$ at which $F$ is increasing in a certain sense. Note that if $x \in F(x)$, then $x \in J$.

Lemma 3.1. Suppose that $F: X \rightarrow X$ satisfies (2) and (3). If $a \in X$ and $M(a) \cap F(a) \neq \varnothing$, then $J \cap M(a) \neq \varnothing$. Hence, $J \neq \varnothing$.

Proof. Let $a \in X$ and let $t_{a} \in F(a) \cap M(a)$ be minimal. Let $K=$ $\left\{p \in\left[a, t_{a}\right]: F(p) \cap\left[p, t_{a}\right] \neq \varnothing\right\}$ and let $x_{0}=\sup K$. For each $p \in K$, select $y_{p} \in F(p) \cap\left[p, t_{a}\right]$, and let $y$ be any $\mathscr{I}$-cluster point of $\left\{y_{p}\right\}$ in $\left[a, t_{a}\right]$. By condition (3), $y \in F\left(x_{0}\right)$. Further, if $p \in K$, then $p \leqq y_{p}$, and so $x_{0} \leqq y$. Let $y_{0}=\inf \left(F\left(x_{0}\right) \cap\left[x_{0}, t_{a}\right]\right)$. By (2), $y_{0} \in F\left(x_{0}\right)$. If $x_{0}=y_{0}$, then $x_{0} \in J \cap M(a)$. So suppose that $x_{0}<y_{0} \leqq t_{a}$. Finally, if $x_{0}<p \leqq y_{0}$, then $F(p) \cap\left[p, y_{0}\right]=\varnothing$. Thus $J \cap M(a) \neq \varnothing$, and so $J \neq \varnothing$ since there is a least element $e \in X$. 
In order to make the proof of the main theorem more readable, we shall prove one more lemma first. For this we need the following sets. For each $x \in J$, set $T(x)$ equal to the associated points $t_{x} \in M(x) \cap F(x)$ and set

$$
\mathscr{J}=\left\{\left(x, t_{x}\right): x \in J \text { and } t_{x} \in T(x)\right\} .
$$

We partially order $\mathscr{J}$ pointwise as follows: $\left(x_{1}, t_{1}\right) \leqq\left(x_{2}, t_{2}\right)$ if and only if $x_{1} \leqq x_{2}$ and $t_{1} \leqq t_{2}$ where $\left(x_{i}, t_{i}\right) \in \mathscr{J}$ for $i=1,2$.

Lemma 3.2. If $F$ satisfies (2) and (3), then $\mathscr{J}$ contains a maximal element.

Proof. By Lemma 3.1, $J$, and hence $\mathscr{J}$, is nonempty. Thus let

$$
\mathscr{C}=\left\{\left(x_{\alpha}, t_{\alpha}\right): \alpha \in \mathscr{A}\right\}
$$

be a chain in $\mathscr{J}$. We may assume that $\left\{x_{\alpha}\right\}$ and $\left\{t_{\alpha}\right\}$ are increasing. Let $x_{0}=\sup _{\alpha} x_{\alpha}$ and $t_{0}=\sup _{\alpha} t_{\alpha}$. By (3), $t_{0} \in F\left(x_{0}\right)$, and since $x_{\alpha} \leqq t_{\alpha} \leqq t_{0}$ for each $\alpha \in \mathscr{A}, x_{0} \leqq t_{0}$. If $x_{0}=t_{0}$, then $x_{0} \in J$ and $\left(x_{0}, t_{0}\right)$ is an upper bound for $\mathscr{C}$ in $\mathscr{J}$. Thus suppose that $x_{0}<t_{0}$. Now if there is a $t \in F\left(x_{0}\right) \cap\left[x_{0}, t_{0}\right]$ such that $x_{0}<t<t_{0}$, then there is an $\alpha \in \mathscr{A}$ such that $t<t_{\alpha}<t_{0}$. But then $x_{\alpha}<x_{0} \leqq t_{\alpha}$ and $F\left(x_{0}\right) \cap\left[x_{0}, t_{\alpha}\right] \neq \varnothing$ which contradicts $x_{\alpha} \in J$. Thus $t_{0}$ is minimal in $F\left(x_{0}\right) \cap M\left(x_{0}\right)$. Now let

$$
P=\left\{p \in\left(x_{0}, t_{0}\right]: F(p) \cap\left[p, t_{0}\right] \neq \varnothing\right\} .
$$

If $P=\varnothing$, then $x_{0} \in J$ and $\left(x_{0}, t_{0}\right)$ is the desired upper bound for $\mathscr{C}$ in $\mathscr{J}$. Thus suppose that $P \neq \varnothing$, and let $p \in P$. Then $F(p) \cap\left[p, t_{0}\right]=\left\{t_{0}\right\}$. For if there exists a $z \in F(p) \cap\left[p, t_{0}\right)$, then there is an $\alpha \in \mathscr{A}$ such that $x_{\alpha}<p \leqq$ $z<t_{\alpha}<t_{0}$. This contradicts the assumption that $x_{\alpha} \in J$. Finally, let $p_{0}=$ sup $P$. Then $p_{0} \in J$ and $\left(p_{0}, t_{0}\right)$ is an upper bound for $\mathscr{C}$ in $\mathscr{J}$. Hence, $\mathscr{J}$ has a maximal element by Zorn's lemma.

In understanding the proof of Lemma 3.2, it is very helpful to look at functions on the unit interval. We are now ready to present our main theorem.

THEOREM 3.3. If $F \in \mathscr{F}$, then $F$ has a fixed point. Hence, $(X, \mathscr{P}, \mathscr{F})$ is a fixed point structure.

Proof. Let $\left(x_{0}, t_{0}\right)$ be maximal in $\mathscr{J}$ and let $C_{0}$ be the chain component of $F\left[x_{0}, t_{0}\right]$ which contains $t_{0}$. If $x_{0}=t_{0}$, then we are done. If $F\left(t_{0}\right) \cap$ $M\left(t_{0}\right) \neq \varnothing$, then $J \cap M\left(t_{0}\right) \neq \varnothing$, which contradicts the maximality of $\left(x_{0}, t_{0}\right)$ in $\mathscr{J}$. Now by (1), $F\left(t_{0}\right) \cap C_{0} \neq \varnothing$. Hence, let $s \in F\left(t_{0}\right) \cap C_{0}$, and set $z_{0}=$ $s \wedge t_{0}$. Note that $z_{0}<t_{0}$ and $z_{0} \in C_{0}$ since $C_{0}$ is a chain component of $F\left[x_{0}, z_{0}\right]$. Next let $p_{0} \in\left(z_{0} \vee x_{0}, t_{0}\right)$. Then $\left[p_{0}, t_{0}\right] \subset C_{0}$, and so each member of $\left[p_{0}, t_{0}\right)$ is in the image of some element of $\left[x_{0}, t_{0}\right]$. Further, if $p_{0} \leqq a<t_{0}$, 
and if $x_{0} \leqq b \leqq t_{0}$ is such that $a \in F(b)$, then $a<b$ since $x_{0} \in J$. Therefore either $F\left(t_{0}\right) \cap\left[p_{0}, t_{0}\right)$ or $F^{-1}\left[p_{0}, t_{0}\right) \cap\left[p_{0}, t_{0}\right)$ is cofinal in $\left[p_{0}, t_{0}\right)$, and thus $t_{0} \in F\left(t_{0}\right)$ by (3).

Before proceeding to a more detailed discussion of the relationships between various classes of multifunctions, we obtain three corollaries which include theorems of Nashef [6] and Ward [8], [9].

Let $X$ be an arboroid. Then by combining the work of Ward [8] with that of Bellamy [1], we see that there is a partial order $\leqq$ for $X$ such that $(X, \leqq)$ is a semitree [5]. Further, each subcontinuum of $X$ is arcwise connected, and so the concepts of refluent and refluent on arcs are equivalent. Further, a closed subset of $X$ satisfies (2), and an u.s.c. point closed multifunction satisfies (3).

Corollary 3.4. If $X$ is an arboroid and if $F: X \rightarrow X$ is u.s.c., point closed, and refluent, then $F$ has a fixed point.

CoRollary 3.5. If $X$ is an arboroid and if $F: X \rightarrow X$ is either an u.s.c. continuum valued multifunction or a continuous, point closed multifunction, then $F$ has a fixed point.

COROLlaRY 3.6. If $X$ is an arcwise connected compact metric space, then $X$ has the fixed point property for u.s.c. point closed refluent multifunctions if and only if $X$ is a dendroid.

We also obtain Young's theorem [10] in a similar fashion. In fact one half of the main fixed point structure in [3] is subsumed by the fixed point structure in the present paper. A technical theorem regarding multifunctions on semitrees is required to see this, and we omit the details. Thus part of Smithson's theorem [7] follows from Theorem 3.3. However, the remainder of the main fixed point structure in [3] is completely independent of Theorem 3.3.

The concept of closed given above defines a topology $\mathscr{T}_{C}$ for a semitree $X$, and the concept of closed under infima for a multifunction $F: X \rightarrow X$ is defined dually to that of closed under suprema. Then it can be proved that $F$ is closed under infima and suprema if and only if the graph of $F$ is closed in $X \times X$, where $X \times X$ has the product topology induced by $\mathscr{T}_{C}$.

In concluding this section, observe that there are multifunctions on the unit interval that satisfy conditions (1)-(3) above and are not u.s.c. Also there are constant multifunctions on the pinched infinite comb [5] that satisfy conditions (1)-(3) above and are not point closed.

4. Refluent multifunctions. A nested Hausdorff space $(X, \mathscr{T})$ admits the structure of a semitree $(X, \leqq)$ in a natural way [5] where $\mathscr{P}$ consists 
of the arcs in $X$. The topology $\mathscr{T}$ is strongly order compatible if $X \backslash M(x)$ and $M(x) \backslash x$ are $\mathscr{T}$ open for all $x \in X$.

THEOREM 4.1. Let $(X, \mathscr{T})$ be a nested Hausdorff space. If one of the following hold:

(a) $\mathscr{T}$ is strongly order compatible;

(b) subcontinua are arcwise connected;

(c) $(X, \mathscr{T})$ is an arboroid;

then the concepts of refluent and refluent on arcs are equivalent for u.s.c. point compact multifunctions.

Proof. If (a) holds, then connected sets are arcwise connected [5].

Fixed point theorems for nested Hausdorff spaces similar to Corollaries 3.4 and 3.5 follow from Theorems 3.3 and 4.1.

Let $\mathscr{F}$ be the set of u.s.c. point compact multifunctions $F: X \rightarrow X$ where $X$ is a nested Hausdorff space. Consider the following subsets of $\mathscr{F}$ :

$C$ - the continuum valued multifunctions in $\mathscr{F}$;

$L$ - the 1.s.c. multifunctions in $\mathscr{F}$;

$L C$ - the multifunctions in $L \cap C$;

$R$-the refluent multifunctions in $\mathscr{F}$;

$R A$ - the multifunctions in $\mathscr{F}$ that are refluent on arcs;

$R A C$ - the multifunctions in $R$ that satisfy: if $x<y$, then every component of $F[x, y]$ is arcwise connected.

The $R A C$ condition was used by Nashef in [6]. The following diagram shows the relationships among the above sets of u.s.c. point compact multifunctions. The solid arrows stand for inclusions in the directions indicated, and the dotted arrows indicate conjectures of the authors.

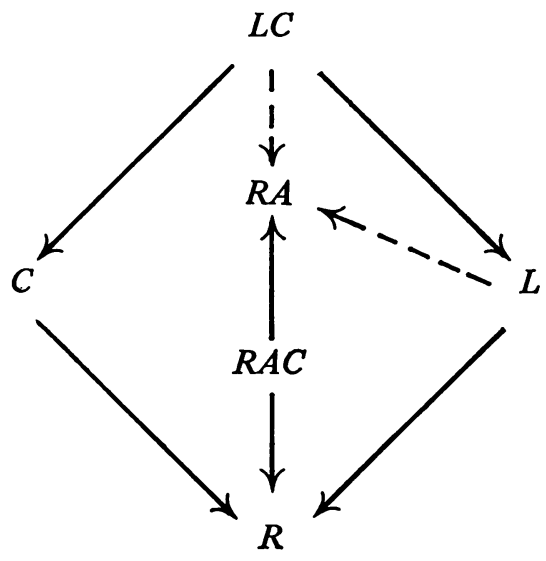

In an arbitrary nested space no inclusion relations hold between any of these sets of multifunctions except those indicated in the above diagram. 
Examples can be found either on the unit interval or the cone over a pseudo arc. For example, let $X$ denote the cone over a pseude arc $A$ with top $e$. Define $F: X \rightarrow X$ by the formula $F(x)=A$ for all $x \in X$. Then $F \in(L C \cap R A) \backslash R A C$. Let $a_{0} \in A$ and define $G: X \rightarrow X$ by the formulas $G(x)=x$ if $x \notin\left[e, a_{0}\right]$, and $G(x)=\{e, x\}$ otherwise. Then $G \in R A \backslash R$. The dotted implications do not hold if the space $X$ is merely required to be arcwise connected or even acyclic.

Establishing the dotted implications in the above diagram would prove a new fixed point theorem for continuous point compact (continuum valued) multifunctions on nested spaces. A natural [9] conjecture would be the following:

Let $X$ be an arcwise connected compact metric space. Then the following are equivalent:

(i) $X$ is nested:

(ii) $X$ has the fixed point property for u.s.c. point closed multifunctions that are refluent on arcs;

(iii) $X$ has the fixed point property for continuous point closed (continuum valued) multifunctions.

Observe that (i) implies (ii) follows from Theorem 3.3 and does not require that $X$ be compact metric.

\section{REFERENCES}

1. D. P. Bellamy, Composants of Hausdorff indecomposable continua; a mapping approach. Pacific J. Math. (to appear).

2. W. Holsztynski, Fixed points of arcwise connected spaces, Fund. Math. 64 (1969), 289-312. MR 40 \#2008.

3. T. B. Muenzenberger and R. E. Smithson, Fixed point structures, Trans. Amer. Math. Soc. 184 (1973), 153-173.

4. - Mobs and nested spaces (to appear).

5. —- The structure of semitrees (to appear).

6. B. En-Nashef, Fixed points for multi-valued functions on B-spaces, Bull. Acad. Polon. Sci. (to appear).

7. R. E. Smithson, Fixed point theorems for certain classes of multifunctions, Proc. Amer. Math. Soc. 31 (1972), 595-600. MR 44 \#5946.

8. L. E. Ward, Jr., A fixed point theorem for multi-valued functions, Pacific J. Math. 8 (1958), 921-927. MR 21 \#2215.

9. - Characterization of the fixed point property for a class of set-valued mappings, Fund. Math. 50 (1961/62), 159-164. MR 24 \#A2956.

10. G. S. Young, Jr., The introduction of local connectivity by change of topology, Amer. J. Math. 68 (1946), 479-494. MR 8, 49.

Department of Mathematics, University of Wyoming, Laramie, Wyoming 82071

Department of Mathematics, University of Houston, Houston, Texas 77004 (Current address of R. E. Smithson)

Current address (T. B. Muenzenberger): Department of Mathematics, Kansas State University, Manhattan, Kansas 66506 